\title{
ALGUNAS DESIGUALDADES EN EL ENVEJECER DE LOS ANCIANOS ESPAÑOLES DE LOS AÑOS NOVENTA
}

\section{Alfredo Alfageme Chao}

Universidad Jaume I de Castellón

\begin{abstract}
RESUMEN
No existe una definición satisfactoria del envejecimiento como proceso universal e irreversible que experimentan todos los individuos. El envejecimiento real u observable viene siendo identificado a partir de sus correlatos convencionales (declive físico, psíquico y social). Utilizando datos españoles de encuesta, se apoya la hipótesis de que las personas relativamente pobres (por razón de su nivel educativo y posición económica) tienden a envejecer peor y más rápido. La mayoría de ellos alcanzan niveles superiores de discapacidad física, aislamiento social y malestar psicológico. Empobrecen, y realmente envejecen, con respecto a las personas mejor situadas socialmente.
\end{abstract}

\section{INTRODUCCIÓN}

$\mathrm{Ni}$ la vejez ni el envejecimiento han llegado a ser definidos de forma satisfactoria. En realidad, sólo alcanzamos a identificar un amplio abanico de variables que actúan «sobre» y "a partir» de la vejez (Fericgla, 1992: 67), o hacemos referencia a diferentes "dimensiones del envejecimiento" (Sánchez Vera, 1997). Un concepto de envejecimiento real u observable (siempre abierto y sólo identificable a partir de sus correlatos físicos, psíquicos y sociales) se distingue de otro concepto hipotético pero ineludible de envejecimiento esencial, inevitable e irreversible, cuya naturaleza última desconocemos (Alfageme, 1998a). 
Se han analizado extensamente una serie de problemas más o menos graves (mala salud, aislamiento social, penuria económica, etc.) que aguardan a muchos de quienes llegan a la jubilación, enviudan o alcanzan edades avanzadas (Bazo, 1990; Rodríguez, 1994). Mucho se ha escrito acerca de la heterogeneidad existente dentro de la población anciana convencional. Sin embargo, pocas veces se adoptan enfoques que permitan investigar las causas de semejantes desigualdades. Ésta es la perspectiva que proponemos aquí.

A falta de mejor acuerdo, podemos afirmar que cualquier factor que provoque o acelere procesos convencionales de declive físico, psíquico y/o social es factor de envejecimiento real u observable (que es el que interesa en la práctica). Así, ciertas desigualdades entre individuos producen diferentes modos y ritmos reales de envejecimiento. Es obvio que estas diferencias no son inevitables y que los procesos no siempre son irreversibles. Con nuestra investigación empírica, apoyaremos la hipótesis de que las personas relativamente pobres (de acuerdo con criterios convencionales de nivel de instrucción e ingresos monetarios), envejecen peor y más rápido (de acuerdo, a su vez, con criterios convencionales de deterioro físico, psíquico y social). Es algo que está en la mente de todos y merece ser abordado con decisión.

Utilizaremos principalmente el estudio sobre «apoyo informal a la tercera edad»(CIS/INSERSO), catalogado por el CIS con el número 2072/1993. Esta encuesta recoge información muy variada, mucha de la cual se adecua bastante bien a nuestros intereses ${ }^{1}$. Las características técnicas de la muestra y el cuestionario pueden encontrarse en INSERSO (1995: 121 y ss.).

Fue posible trabajar, además de otros aspectos menos elaborados, con dos importantes dimensiones de desigualdad que van a protagonizar el análisis: pobreza/riqueza, por una parte, y discapacidad física, por otra. Se procuró que las medidas fueran capaces de sugerir, con cierto rigor, información pretérita o retrospectiva acerca de los individuos, aun a costa de reducir su contenido. A tal efecto, se empleó un «índice retrospectivo de desigualdad» (elaborado básicamente a partir del nivel de instrucción y del importe de la pensión que perciben los encuestados) y un "índice de discapacidad alcanzada» (construido a partir de algunos indicadores sobre la capacidad de los ancianos para la realización de actividades cotidianas y de su estado de salud). Ambos índices son presentados con una puntuación de 0 a 10 puntos y los individuos eventualmente agrupados por intervalos ${ }^{2}$.

${ }^{1}$ La encuesta del CIS deriva de una colaboración con el INSERSO. Éste último ha publicado ya los principales resultados de la misma (INSERSO, 1995). Nuestro enfoque, como se verá, es netamente complementario al adoptado por el INSERSO, en cuya introducción se considera preciso continuar abordando el estudio de las personas mayores dependientes. Entre otras cosas — se propone-, será bueno relacionar los niveles de necesidad de ayuda con otras dimensiones como el status socioeconómico y el grado de apoyo social disponible (Rodríguez, 1995: 23).

${ }^{2}$ El procedimiento detallado de construcción de estos índices, así como una presentación más extensa de los resultados que siguen, puede verse en Alfageme (1997). Muy resumidamente, el índice retrospectivo de desigualdad ha sido elaborado a partir de factores (instrucción e ingre- 


\section{DESIGUALDAD EN CUANTO A DISCAPACIDAD ALCANZADA}

Las desigualdades experimentadas a lo largo de la vida influyen en el grado de discapacidad alcanzada por las personas según van envejeciendo. Rechazamos de entrada la posibilidad de un análisis de correlaciones, dado que éste sólo es aplicable a variables que presentan una cierta normalidad en su distribución de frecuencias. Este requisito se podría dar por cumplido en el caso del índice retrospectivo de desigualdad. Sin embargo, el índice de discapacidad alcanzada presenta una gran desviación hacia la izquierda, merced al saludable estado general de nuestros mayores ${ }^{3}$. Optamos entonces por analizar los resultados acudiendo al empleo de intervalos operativos, valores medios y porcentajes.

\section{Comprobando la influencia de cada variable}

Vemos, primeramente, que las dos variables que componen el índice retrospectivo de desigualdad influyen de forma notable en el grado de discapacidad (Tabla 1). El índice medio de discapacidad se triplica, aproximadamente, entre las categorías extremas de nivel de instrucción e ingresos. Observamos, de paso, que el valor correspondiente a la categoría de casos perdidos de ingresos es muy cercano a la media. Esto sugiere que tales casos estarían bien repartidos entre el resto de categorías y no deberían pertubar significativamente el análisis ${ }^{4}$.

sos) que se supone resumen múltiples aspectos de la vida pasada de los individuos. Por su parte, para la elaboración del índice de discapacidad alcanzada se ha tenido en cuenta, principalmente, la capacidad declarada por los encuestados para la realización de una serie de actividades elementales (vestirse, comer, caminar, etc.). Se supone que la mayoría de quienes tienen problemas para realizar tales actividades no los tuvieron anteriormente, es decir, que han experimentado un proceso de deterioro al respecto. Las tablas 1 y 2 muestran las variables que componen ambos índices. En posteriores tablas aparecen los individuos agrupados por intervalos operativos. Así, el estrato "rico» incluye aquellos individuos con puntuación superior a 7 en índice retrospectivo de desigualdad (26,5 por 100 de los casos), el estrato «medio» a los que puntúan entre 3 y 7 (48,5 por 100) y el estrato "pobre» a los que se sitúan por debajo de 3 (25,0 por 100). Por su parte, el grupo de «discapacitados» recoge a los individuos con puntuación superior a 2 en índice de discapacidad alcanzada (26,2 por 100 de los casos), considerándose «capacitados» al resto $(73,8$ por 100).

${ }^{3}$ No obstante, el coeficiente de correlación entre el índice retrospectivo de desigualdad y el índice de discapacidad alcanzada es de 0,28 para la totalidad de la muestra, ascendiendo a 0,32 tras aplicar una transformación logarítmica (que mejora ligeramente la normalidad de su distribución de frecuencias) al índice de discapacidad alcanzada. Esto sugiere ya, aunque de forma muy burda, una relación positiva entre ambas variables.

${ }^{4}$ Para estos casos perdidos de ingresos (poco más de un 12 por 100 sobre el total), el índice retrospectivo de desigualdad se construye exclusivamente a partir del nivel de instrucción. 


\section{TABLA 1}

\section{Indice medio de discapacidad alcanzada (I) y número de casos sin ponderar (N) para cada categoría de nivel de estudios y nivel de ingresos \\ (Españoles mayores de 64 años)}

\begin{tabular}{|c|c|c|}
\hline & $I$ & $(N)$ \\
\hline \multicolumn{3}{|l|}{ Nivel de estudios } \\
\hline 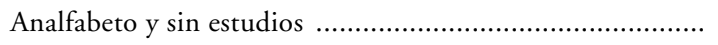 & 2,87 & $(329)$ \\
\hline 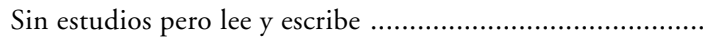 & 2,07 & $(523)$ \\
\hline 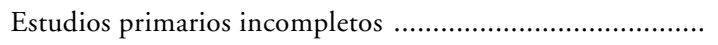 & 1,84 & $(449)$ \\
\hline 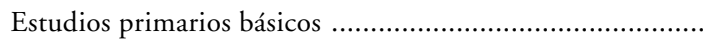 & 1,44 & $(806)$ \\
\hline 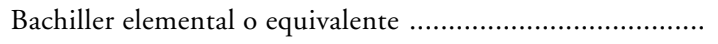 & 1,23 & $(172)$ \\
\hline Bachiller superior o equivalente ............................ & 0,87 & $(50)$ \\
\hline 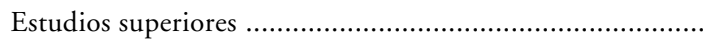 & 0,71 & (63) \\
\hline \multicolumn{3}{|l|}{ Ingresos mensuales individuales } \\
\hline Menos de 25.000 ptas. ............. & 3,13 & $(55)$ \\
\hline De 25.000 a 35.000 ptas. ................. & 2,43 & $(135)$ \\
\hline De 35.000 a 45.000 ptas. ............... & 2,58 & $(268)$ \\
\hline 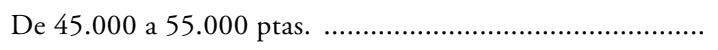 & 1,91 & $(699)$ \\
\hline 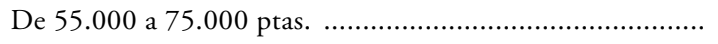 & 1,49 & $(456)$ \\
\hline 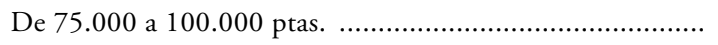 & 1,41 & $(312)$ \\
\hline 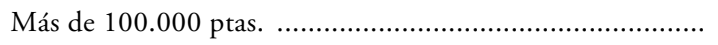 & 1,09 & $(185)$ \\
\hline 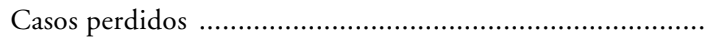 & 1,82 & $(309)$ \\
\hline
\end{tabular}

FUENTE: CIS, estudio núm. 2072, 1993 (elab. propia).

Realizamos ahora el ejercicio inverso. La desigualdad retrospectiva, presentada por intervalos operativos, afecta significativamente a cada una de las variables que componen el índice de discapacidad alcanzada (Tabla 2). La relación se cumple sin excepción. Los diez aspectos de la discapacidad aumentan sus puntuaciones medias al pasar del estrato rico al medio y de éste al pobre. Debe quedar claro que la desigualdad en cuanto a discapacidad alcanzada es más amplia de lo que sugieren los intervalos operativos propuestos 5 .

5 El índice global de discapacidad pasa de un valor medio de 1,05 para el estrato rico a 2,56 para el estrato pobre (Tabla 4). Añadimos que el mismo índice de discapacidad desciende hasta 0,69 para los individuos con puntuación menor o igual que 1 en índice retrospectivo de desigualdad (sobre 65 casos sin ponderar), y asciende a una media de 3,03 para los individuos con puntuación superior a 9 (154 casos). Si mantenemos aquellos intervalos más amplios es por la necesidad de controlar por otras variables que señalamos seguidamente. 
En lo sucesivo, trabajaremos con los índices globales, pues su presentación desglosada obligaría a construir una gran cantidad de tablas que aportaría poco al objetivo central del análisis.

\section{TABLA 2}

Puntuaciones medias en discapacidad para la realización de actividades cotidianas y por razón de algunos indicadores del estado de salud, para cada categoría de indice retrospectivo de desigualdad

(Españoles mayores de 64 años)

\begin{tabular}{|c|c|c|c|c|}
\hline & \multicolumn{3}{|c|}{ Indice retrospectivo de desigualdad } & \multirow[b]{2}{*}{$\begin{array}{c}\text { Todos } \\
(2.465)\end{array}$} \\
\hline & $\begin{array}{c}\text { Estrato rico } \\
\text { (617) }\end{array}$ & $\begin{array}{c}\text { Estrato medio } \\
(1.195)\end{array}$ & $\begin{array}{c}\text { Estrato pobre } \\
\text { (653) }\end{array}$ & \\
\hline \multicolumn{5}{|l|}{ Actividades cotidianas: } \\
\hline Levantarse de la cama ........................ & 0,52 & 1,11 & 1,73 & 1,13 \\
\hline Vestirse o desvestirse .......................... & 0,47 & 1,16 & 1,95 & 1,20 \\
\hline Bañarse o ducharse ............................. & 0,85 & 1,78 & 3,05 & 1,88 \\
\hline 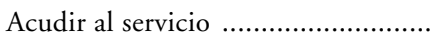 & 0,30 & 0,81 & 1,43 & 0,85 \\
\hline 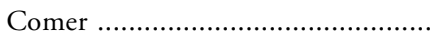 & 0,20 & 0,49 & 1,02 & 0,56 \\
\hline Subir o bajar escaleras ..................... & 1,07 & 2,02 & 3,07 & 2,07 \\
\hline 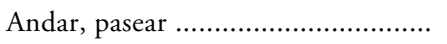 & 0,77 & 1,62 & 2,60 & 1,67 \\
\hline \multicolumn{5}{|l|}{ Indicadores de salud: } \\
\hline Estado general declarado .................. & 3,66 & 4,49 & 5,43 & 4,54 \\
\hline Estado de la vista .......................... & 1,66 & 2,35 & 3,35 & 2,44 \\
\hline 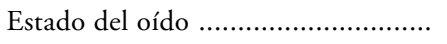 & 1,16 & 1,44 & 2,36 & 1,62 \\
\hline Índice de discapacidad alcanzada .... & 1,05 & 1,72 & 2,56 & 1,78 \\
\hline
\end{tabular}

FUENTE: CIS, estudio núm. 2072, 1993 (elaboración propia).

\section{Controlando por sexo y edad}

Sabemos que la discapacidad aumenta estadísticamente con la edad. Y sabemos que - en promedio - tanto el nivel de instrucción como la cuantía de las pensiones son inferiores entre los ancianos de más edad (por razón de los efectos de cohorte y devaluación de las pensiones). Es muy conveniente, si no necesario, relacionar los índices controlando por la edad. También conviene 
tener en cuenta el sexo. Las mujeres tienden a mostrar un peor estado de salud (a pesar de que viven más años) y han llevado en general una vida más confinada al hogar.

La tabla 3 ofrece la presentación más completa y rigurosa a la que hemos podido llegar. Muestra el índice de discapacidad alcanzada para cada categoría operativa de índice retrospectivo de desigualdad, sexo y edades. Con el fin de poder valorar adecuadamente la significatividad de los resultados, presentamos los números de casos (sin ponderar) para cada casilla.

La parte superior de la tabla 3 controla por edad (grupos quinquenales) y sexo. La discapacidad alcanzada, en todos los casos, disminuye con la riqueza y aumenta con la pobreza. Esta regularidad es, quizá, lo más destacable. La serie de valores concretos estaría más abierta a variaciones, en función de los componentes de los índices y de los intervalos utilizados. Se observa también la mayor discapacidad alcanzada por las mujeres en casi todos los casos, especialmente los de 80 y más años de edad. Ello concuerda con los resultados de otras investigaciones e inspira confianza en estos datos.

La parte inferior de la misma tabla 3 ofrece un aconsejable control exhaustivo por la edad (grupos bianuales). Con las únicas excepciones de los estratos ricos de «87 y 88 años» y «89 y más» (que incluyen las insignificantes cifras de 6 y 5 casos, respectivamente, y entendemos que no deben ser tenidos en cuenta), las previsiones se continúan cumpliendo con gran regularidad. Parece claro que, a cualquier edad, los que han sido y son más pobres se han discapacitado en considerable mayor grado que los que fueron y son más ricos (hablamos, obviamente, en términos medios).

Justo es comentar también el notable poder explicativo que, en términos estrictamente estadísticos, tiene la edad cronológica. A mayor edad, mayor discapacidad. Esto no sorprende a nadie. El reconocimiento de la universalidad e irreversibilidad del proceso de envejecimiento es suficiente para dar razón de ello. Por otra parte, el poder explicativo de la edad no presenta una total regularidad. Pasados los 65 ó 66 años —superado, cabe interpretar, el tan citado trauma de la jubilación- los índices medios de discapacidad alcanzada se reducen ligeramente. La tendencia normal al aumento reaparece a edades más avanzadas. Esto es visible en la parte inferior de la tabla 3. Más difícil es la interpretación de otra serie de irregularidades que aparecen tanto en el estrato pobre como, muy especialmente, en el estrato rico. En general, los cursos vitales de unos y otros (los más ricos y los más pobres) pueden diferir en muchos sentidos de la «normalidad» de las clases medias. Es más probable que aparezcan irregularidades a cualquier edad. 


\section{TABLA 3}

Indice medio de discapacidad alcanzada (I) y número de casos sin ponderar (N) para cada categoría de indice retrospectivo de desigualdad, sexo y edades (Españoles mayores de 64 años)

\begin{tabular}{|c|c|c|c|c|c|c|c|c|}
\hline & \multicolumn{6}{|c|}{ Indice retrospectivo de desigualdad } & & \\
\hline & \multicolumn{2}{|c|}{ Estrato rico } & \multicolumn{2}{|c|}{ Estrato medio } & \multicolumn{2}{|c|}{ Estrato pobre } & \multicolumn{2}{|c|}{ Todos } \\
\hline & $I$ & $(N)$ & $I$ & $(N)$ & $I$ & $(N)$ & $I$ & $(N)$ \\
\hline \\
\hline Varón ........ & 0,86 & $(283)$ & 1,53 & $(483)$ & 2,27 & $(184)$ & 1,44 & $(950)$ \\
\hline 65 a 69 años & 0,58 & (92) & 1,14 & $(107)$ & 1,78 & $(26)$ & 0,98 & $(225)$ \\
\hline 70 a 74 años .... & 0,71 & (70) & 1,18 & $(101)$ & 1,56 & (34) & 1,08 & $(205)$ \\
\hline 75 a 79 años. & 1,64 & (82) & 1,70 & $(149)$ & 2,42 & (53) & 1,82 & (284) \\
\hline 80 y más años & 1,45 & (39) & 2,68 & $(126)$ & 3,22 & (71) & 2,64 & $(236)$ \\
\hline Mujer & 1,28 & $(250)$ & 1,86 & $(676)$ & 2,67 & $(510)$ & 2,01 & $(1.436)$ \\
\hline 65 a 69 años & 1,06 & (68) & 1,20 & $(129)$ & 1,30 & $(63)$ & 1,19 & $(260)$ \\
\hline 70 a 74 años & 0,92 & (69) & 1,51 & $(122)$ & 2,29 & (84) & 1,60 & $(275)$ \\
\hline 75 a 79 años & 1,64 & (65) & 1,80 & $(204)$ & 2,54 & $(167)$ & 2,06 & $(436)$ \\
\hline 80 y más años ............ & 2,54 & $(48)$ & 3,25 & $(221)$ & 4,19 & (196) & 3,57 & $(465)$ \\
\hline \multicolumn{9}{|l|}{ Ambos sexos } \\
\hline 65 a 69 años ..... & 0,78 & $(160)$ & 1,17 & $(236)$ & 1,44 & (89) & 1,09 & $(485)$ \\
\hline 70 a 74 años ..... & 0,82 & (139) & 1,36 & $(223)$ & 2,08 & (118) & 1,38 & $(480)$ \\
\hline 75 a 79 años ..... & 1,64 & (147) & 1,76 & $(353)$ & 2,51 & $(220)$ & 1,96 & $(720)$ \\
\hline 80 y más años ...... & 2,05 & $(87)$ & 3,04 & $(347)$ & 3,93 & $(267)$ & 3,26 & $(701)$ \\
\hline \multicolumn{9}{|l|}{ Edad (bianual) } \\
\hline 65 y 66 años & 0,86 & (69) & 1,27 & (94) & 1,42 & $(32)$ & 1,15 & $(195)$ \\
\hline 67 y 68 años. & 0,74 & (63) & 1,11 & (89) & 1,43 & (31) & 1,04 & (183) \\
\hline 69 y 70 años.. & 0,76 & (57) & 1,21 & $(106)$ & 1,53 & (54) & 1,17 & $(217)$ \\
\hline 71 y 72 años.. & 0,64 & (49) & 1,28 & $(101)$ & 2,12 & (43) & 1,31 & (193) \\
\hline 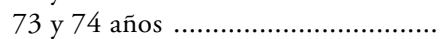 & 0,93 & (61) & 1,49 & $(69)$ & 2,33 & (47) & 1,52 & $(177)$ \\
\hline 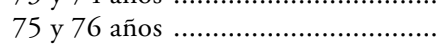 & 1,50 & (79) & 1,60 & $(152)$ & 1,98 & (75) & 1,67 & $(306)$ \\
\hline 77 y 78 años & 1,87 & $(50)$ & 1,94 & $(124)$ & 2,61 & (86) & 2,15 & $(260)$ \\
\hline 79 y 80 años. & 1,64 & (36) & 1,94 & $(131)$ & 3,14 & (89) & 2,31 & $(256)$ \\
\hline 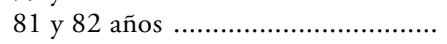 & 1,55 & (23) & 2,45 & $(84)$ & 3,75 & (51) & 2,74 & $(158)$ \\
\hline 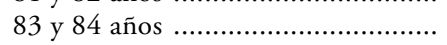 & 0,96 & (19) & 2,88 & $(72)$ & 3,28 & (68) & 2,82 & $(159)$ \\
\hline 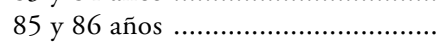 & 2,47 & $(16)$ & 3,20 & $(58)$ & 3,84 & (39) & 3,32 & $(113)$ \\
\hline 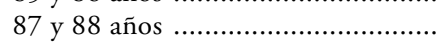 & 3,93 & $(6)$ & 3,82 & (33) & 4,42 & $(31)$ & 4,09 & $(70)$ \\
\hline 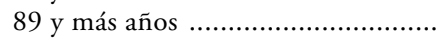 & 6,26 & $(5)$ & 4,65 & $(46)$ & 5,17 & $(48)$ & 4,98 & $(99)$ \\
\hline & 1,05 & & 1,72 & 159) & 2,56 & 694) & & \\
\hline
\end{tabular}

FUENTE: CIS, estudio núm. 2072, 1993 (elaboración propia). 


\section{Desigualdad, discapacidades y minusvalias}

Insertamos ahora alguna información procedente de la encuesta del INE sobre «discapacidades, deficiencias y minusvalías» (INE, 1987a). El índice de discapacidad alcanzada con el que venimos operando no sugiere simplemente "discapacidad» sino también, al menos en parte, "minusvalía». La OMS propone útiles definiciones de estos conceptos ${ }^{6}$.

La tabla 4, referida a toda la población española, confirma que tanto las discapacidades como las minusvalías disminuyen a un mayor nivel de instrucción (referido aquí a la persona principal del hogar) y de ingresos familiares. Lo que más quisiéramos destacar es que también disminuye en el mismo sentido la proporción de personas con discapacidades que, además, padecen minusvalías o cuya discapacidad —en otras palabras - implica para ellos alguna minusvalía (columna derecha de la Tabla 4).

Como se indica en una publicación del propio INE (1987b: 85), «no todas las personas que padecen determinadas discapacidades han de tener necesariamente alguna minusvalía puesto que, por su propia naturaleza, algunas limitaciones no impiden a la persona comportarse de acuerdo con las normas o costumbres que rigen la sociedad, y porque la discapacidad padecida puede haber sido superada de forma que no inhabilite a la persona en su relación social». Así, la mayor aparición de minusvalías entre las personas con discapacidades, según disminuye su instrucción e ingresos, puede deberse tanto al hecho de que las discapacidades sean más graves como a una menor disponibilidad de recursos de muy diverso tipo que permitan su superación a nivel social. Es el binomio discapacidad-barreras, y no uno sólo de sus componentes, el que produce minusvalía. Y las barreras tienen que ver con un variado repertorio de recursos individuales y comunitarios.

Nos parece que es más relevante, para la calidad de vida, la minusvalía que la discapacidad. E interpretamos que el comportamiento del índice de discapacidad alcanzada en función del índice retrospectivo de desigualdad (Tabla 3) no sólo refleja la mayor discapacidad (según la definición de la OMS) alcanzada por los más pobres. Refleja también el hecho de que tal discapacidad se supera mejor — se elude en mayor medida la aparición de minusvalías — si se dispone de recursos adecuados. Las posibilidades de entrar en círculos viciosos son claras y variadas. A menor disponibilidad de recursos (de diverso tipo), mayor probabilidad de padecer discapacidades y de que éstas supongan minusvalías. A mayor minusvalía, por su parte, mayor probabilidad de empeoramiento en cuanto a discapacidad y, en general, de empobrecimiento en

6 Una «deficiencia» es cualquier pérdida o anomalía de un órgano o de la función propia de este órgano. «Discapacidades» son las consecuencias que la deficiencia produce a nivel de la persona. Las "minusvalías», finalmente, se refieren a las consecuencias producidas a nivel social, es decir, las desventajas originadas en la relación del individuo con su entorno social (costumbres, comportamientos y normas que impone la sociedad). Con estos conceptos trabaja expresamente la encuesta del INE (INE, 1987a: 7). 


\section{TABLA 4}

Porcentajes de personas con discapacidades y con minusvalias (sobre la población total española) y porcentaje de personas con minusvalias sobre el total de personas con discapacidades (conversión), seguin el nivel de estudios de la persona principal del hogar y nivel de ingresos familiares

\begin{tabular}{ccc} 
Porcentaje & Porcentaje & Porcentaje \\
de personas & depersonas & de conversión \\
con discapacidades & con minusvalias & (minus./discap.) \\
\hline
\end{tabular}

Nivel de estudios de la persona principal:

Analfabetos

30,7

Sin estudios

20,8

14,0

45,6

$1 .^{\text {er }}$ grado

12,4

8,6

41,3

$2 .^{\circ}$ grado (1. ${ }^{\text {er }}$ ciclo)

8,4

4,8

38,7

$2 .^{\circ}$ grado (2. ${ }^{\circ}$ ciclo)

7,1

3,2

38,1

$3 .^{\text {er }}$ grado ( $1 .^{\text {er }}$ ciclo $)$

7,4

2,4

33,8

$3 .^{\text {er }}$ grado $\left(2 .^{\circ}\right.$ ciclo $)$

6,4

2,3

31,1

2,0

31,2

Ingresos familiares (pesetas/mes):

Menos de 15.000

18,7

9,4

50,3

15.000 a 30.000

26,7

10,9

30.000 a 50.000

20,1

8,2

12,1

5,0

40,8

50.000 a 75.000

9,2

3,4

40,8

75.000 a 100.000

7,8

2,7

41,3

100.000 a 150.000

6,6

2,1

37,0

150.000 a 200.000

6,1

34,6

200.000 a 300.000

4,9

31,8

Más de 300.000

1,6

26,2

Todos

15,0

1,2

24,5

FUENTE: INE, Encuesta sobre discapacidades, deficiencias y minusvalías, 1986 (elaboración propia).

muchos e interrelacionados aspectos (por incapacidad laboral, disminución de las relaciones sociales, deterioro psíquico, reducción de actividades, dificultades de acceso a la información, etc.).

\section{Un sugerente asomo al mundo rural}

La tabla 5 presenta los índices medios de discapacidad alcanzada en función de los intervalos de desigualdad retrospectiva, controlando por tamaño del municipio de residencia. La hipótesis principal (a mayor pobreza, mayor discapacidad) se cumple en todos los casos. 


\section{TABLA 5}

Indice medio de discapacidad alcanzada (I) y número de casos sin ponderar (N) para cada categoría de indice retrospectivo de desigualdad y tamaño del municipio de residencia

(Españoles mayores de 64 años)

\begin{tabular}{|c|c|c|c|c|c|c|c|c|}
\hline & \multicolumn{6}{|c|}{ Indice retrospectivo de desigualdad } & & \\
\hline & \multicolumn{2}{|c|}{ Estrato rico } & \multicolumn{2}{|c|}{ Estrato medio } & \multicolumn{2}{|c|}{ Estrato pobre } & \multicolumn{2}{|c|}{ Todos } \\
\hline & $I$ & $(N)$ & $I$ & $(N)$ & $I$ & $(N)$ & $I$ & $(N)$ \\
\hline Número de habitantes del municipio: & & & & & & & & \\
\hline Menos de 2.000 & 1,38 & (33) & 1,61 & $(122)$ & 2,16 & (71) & 1,74 & (226) \\
\hline 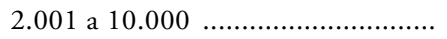 & 1,27 & $(65)$ & 1,77 & $(221)$ & 2,41 & $(139)$ & 1,88 & $(425)$ \\
\hline 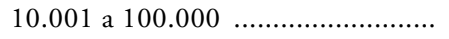 & 1,06 & $(147)$ & 1,89 & $(345)$ & 2,62 & $(285)$ & 1,94 & (777) \\
\hline 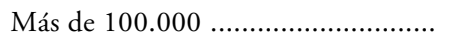 & 0,97 & $(289)$ & 1,60 & $(475)$ & 2,75 & $(200)$ & 1,61 & $(964)$ \\
\hline Todos & 1,05 & $(534)$ & 1,72 & 1.163) & 2,56 & $(695)$ & 1,77 & 2.0 \\
\hline
\end{tabular}

FUENTE: CIS, estudio núm. 2072, 1993 (elaboración propia).

Por otra parte, las diferencias en cuanto a discapacidad alcanzada por «ricos" y "pobres» son sensiblemente mayores cuando se trata de residentes en municipios de mayor tamaño. Tanto es así que la discapacidad media alcanzada, para los estratos ricos, tiende a disminuir a medida que aumenta el tamaño del municipio, mientras que, para los estratos pobres, ocurre lo contrario.

El medio rural — cabe deducir- es más igualitario en cuanto a procesos de discapacitación. En la gran ciudad se cumple con más claridad que los que son más ricos o pobres por razón de sus niveles de instrucción e ingresos lo son también por razón de su discapacidad alcanzada. En este sentido, la ciudad favorece a los ricos y perjudica a los pobres. Encontramos aquí motivos para creer que el capitalismo genera desigualdades y las hace más grandes ${ }^{7}$.

La cuestión, sin embargo, está lejos de ser sencilla. En el medio rural — cuya extraordinaria diversidad difícilmente viene representada por la simple

Como apunta Tortosa (1993: 105), el capitalismo prefiere la explotación agraria para el mercado, aunque genere pobreza, a la explotación para el autoconsumo, aunque sirva de paliativo para la pobreza. A pesar de ello, persisten en el campo español importantes niveles de este segundo tipo de explotación, en el ámbito de la agricultura familiar. Se entiende que incluso las familias más pobres tienen acceso a algún pedazo de tierra, o participan en redes vecinales de circulación de productos, comportamientos propiciados por un modo de vida rural relativa y característicamente solidario. Si a ello unimos la tradicional convivencia intergeneracional propia del medio rural, no habrá de extrañarnos que la pobreza allí sea menos dramática que en el medio urbano. 
indicación del tamaño del municipio-, la pobreza atraviesa otras dimensiones. Entre éstas se encuentra el carácter deprimido y marginado de algunas zonas, de lo cual no es posible dar mínima cuenta aquí. La encuesta del CIS, como la mayoría, es básicamente urbana.

\section{DESIGUALDAD EN CUANTO A AISLAMIENTO SOCIAL}

Vamos a trabajar ahora con algunos indicadores que, de forma bastante directa o convencional, sugieren algún tipo de aislamiento social. Lo que sigue contribuirá a comprender mejor lo que precede. Se reconoce hoy que la discapacidad y la salud, desde puntos de vista físico-biológicos, no son ajenas al bienestar psicológico y social.

\section{Viudez y soledad}

La mayoría de nuestros ancianos vivieron en pareja durante muchos años y, de sobrevivir ambos cónyuges, continúan haciéndolo en la inmensa mayoría de los casos. Es la viudez, sin embargo, factor característico de soledad, aislamiento y empobrecimiento en la última etapa de la vida, evento que experimentan más mujeres que varones merced a su mayor longevidad media ${ }^{8}$. Procede entonces analizar las formas de convivencia de los viudos (mujeres en un alto porcentaje), que relacionaremos aquí con las categorías de índice retrospectivo de desigualdad.

La tabla 6 ofrece una relación de este tipo para cada categoría, a su vez, de índice de discapacidad alcanzada. Este control es muy conveniente puesto que - como sabemos - el declive físico es una de las razones por las que los ancianos, especialmente si han enviudado, son acogidos y cuidados por otras personas (sus hijos principalmente).

Vemos que, entre los viudos capacitados, domina tanto la soledad como la convivencia con los hijos, mientras que entre los discapacitados se impone claramente la segunda de estas formas. Se confirma la extendida tendencia al acogimiento de los ancianos por parte de sus hijos cuando aquéllos padecen discapacidades y han perdido a su cónyuge. No hay diferencias significativas entre ricos y pobres en cuanto a la proporción de convivientes con sus hijos, salvo una cierta tendencia de los más ricos y capacitados a no vivir de esta manera (debido, probablemente, a su mayor autonomía).

${ }^{8}$ No analizaremos diferencias por razón del sexo porque no lo permite el tamaño de la muestra (sólo un 20 por 100 de los viudos/as encuestados por el CIS son varones). Se ha sugerido a menudo (Alfageme, 1998b) que la mayor soledad femenina parece relacionada tanto con las desigualdades en cuanto a esperanza de vida como con una mayor autonomía por parte de las mujeres, y no porque sus hijos u otras personas estén menos dispuestos a convivir con ellas. 


\section{TABLA 6}

Forma de convivencia según indice de discapacidad alcanzada e indice retrospectivo de desigualdad (porcentajes verticales)

(Españoles viudos mayores de 64 años)

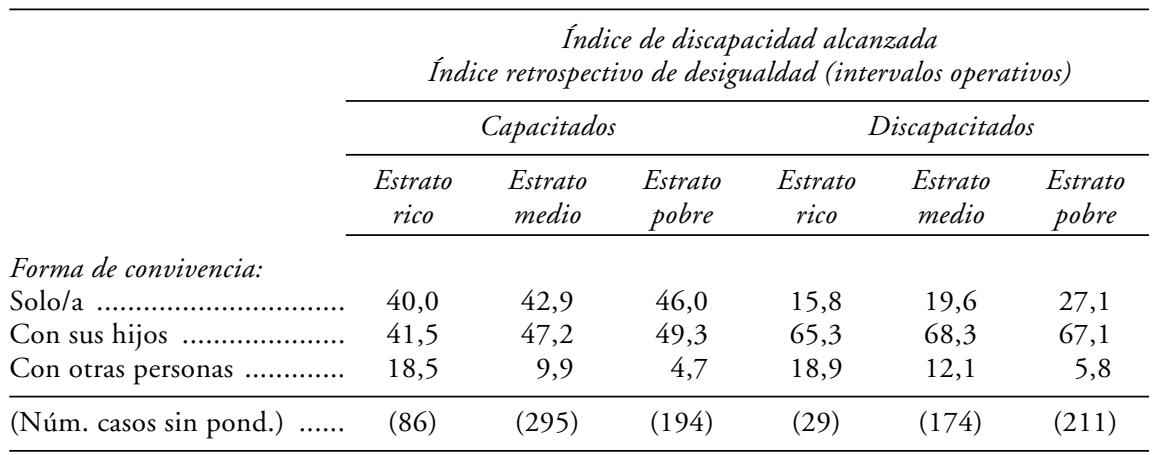

FUENTE: CIS, estudio núm. 2072, 1993 (elaboración propia).

Sin embargo, la soledad es más corriente en los estratos pobres, siendo bastante más significativo este contraste entre ricos y pobres dentro del grupo de discapacitados. La minoritaria soledad de los ricos se compensa claramente con unos mayores porcentajes de convivientes «con otras personas» (con familiares que no sean sus hijos, con nuevas parejas, con empleado/a del hogar, etc.). Los ricos, por tanto, tienden a diversificar más su forma de convivencia, debido probablemente a sus más amplias posibilidades de compensar (económicamente o de otra manera) a sus eventuales acompañantes. Por su parte, los pobres que por la razón que sea no viven con sus hijos se ven abocados a la soledad en notable mayor proporción. Resumiendo, podemos decir que si la soledad es factor de pobreza (afirmación más extendida y convincente), también la pobreza - en confluencia con la viudez- es factor de soledad. Los problemas tienden a acumularse en cualquiera de los dos sentidos.

Con todo, hemos de advertir que la soledad domiciliaria no pasa de ser un indicador poco riguroso de aislamiento social. Se trata, en realidad, de un factor de riesgo. El aislamiento social es un problema complejo, objeto muy indicado de análisis cualitativos. Tan solo sugerimos aquí algunos apuntes cuantitativos de muy discutible significación.

\section{Aislamiento respecto al entorno social más amplio}

Las personas, los ancianos en particular, pueden gozar de la cercanía de unos pocos familiares, vecinos o amigos, permaneciendo su vida social confinada -ésta es la cuestión ahora- a pequeños círculos microgrupales de este tipo. 
La tabla 7 muestra que la frecuencia con que se realizan distintas actividades sociales (desde las más frecuentes, como "ver la televisión» u "oír la radio", hasta las más minoritarias, como «hacer algún deporte») es mayor entre los ricos que entre los pobres. También es mayor, lo que es más obvio, entre los capacitados.

El contraste más espectacular es el correspondiente a la actividad «leer», lo cual es muy lógico considerando que el nivel de instrucción es el principal componente del índice retrospectivo de desigualdad. Se ha insistido mucho en que usar la capacidad intelectual es lo mejor que se puede hacer para no perderla. Toda actividad - la lectura, entre otras, característicamente- implica un uso de la capacidad intelectual. Por otra parte, casi todas las actividades, excepto, lamentablemente, las más realizadas (como "ver la televisión») suponen un saludable ejercicio físico. De nuevo, el círculo vicioso de empobrecimiento y de envejecimiento amenaza a los más desfavorecidos.

También los más pobres van menos «al bar, cafetería» y salen menos para «hacer compras, recados», actividades para las que hace falta dinero. Pero incluso entre ellos son menos los que suelen «ir al parque, pasear» y «acudir a un club, sociedad». Parece que en el grado de actividad pudiera influir un relativo desánimo por parte de los más desfavorecidos, así como su mayor ignorancia acerca de los beneficios que una vida activa supone en cuanto al estado de salud física y psíquica. Muchos procesos perjudiciales muestran un carácter acumulativo.

\section{TABLA 7}

\section{Porcentajes de personas que realizan diversas actividades con frecuencia semanal o superior, para cada categoría de indice de discapacidad alcanzada e indice retrospectivo de desigualdad \\ (Españoles mayores de 64 años)}

\begin{tabular}{|c|c|c|c|c|c|c|}
\hline & \multicolumn{6}{|c|}{$\begin{array}{l}\text { Indice de discapacidad alcanzada } \\
\text { Indice retrospectivo de desigualdad }\end{array}$} \\
\hline & \multicolumn{3}{|c|}{ Capacitados } & \multicolumn{3}{|c|}{ Discapacitados } \\
\hline & $\begin{array}{c}\text { Estrato } \\
\text { rico } \\
(524) \\
\end{array}$ & $\begin{array}{c}\text { Estrato } \\
\text { medio } \\
(878) \\
\end{array}$ & $\begin{array}{c}\text { Estrato } \\
\text { pobre } \\
(367)\end{array}$ & $\begin{array}{c}\text { Estrato } \\
\text { rico } \\
(83) \\
\end{array}$ & $\begin{array}{c}\text { Estrato } \\
\text { medio } \\
(280) \\
\end{array}$ & $\begin{array}{c}\text { Estrato } \\
\text { pobre } \\
\text { (263) }\end{array}$ \\
\hline \multicolumn{7}{|l|}{ Actividades: } \\
\hline Leer & 78 & 55 & 27 & 46 & 29 & 12 \\
\hline 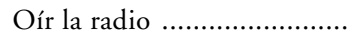 & 75 & 72 & 62 & 55 & 51 & 51 \\
\hline Ver la televisión . ..................... & 97 & 95 & 90 & 94 & 84 & 82 \\
\hline Ir al bar, cafetería ................... & 31 & 22 & 19 & 11 & 9 & 8 \\
\hline Ir al parque, pasear ................ & 69 & 61 & 45 & 35 & 36 & 30 \\
\hline Hacer compras, recados ....... & 74 & 75 & 64 & 39 & 31 & 27 \\
\hline Ir a un club, sociedad .......... & 22 & 20 & 12 & 11 & 10 & 6 \\
\hline Hacer algún deporte ............. & 12 & 6 & 4 & 0 & 1 & 0 \\
\hline
\end{tabular}

FUENTE: CIS, estudio núm. 2072, 1993 (elaboración propia). 
No deben ya sorprender, finalmente, los resultados contenidos en la tabla 8. Entre los más pobres - siempre de acuerdo con las categorías operativas que venimos manejando-, es inferior el porcentaje de individuos que conocen o saben de la existencia de una serie de servicios públicos y prestaciones sociales típicamente dirigidos a la Tercera Edad?.

\section{TABLA 8}

Porcentajes de personas que conocen o saben de la existencia de diversos servicios públicos y prestaciones sociales para la Tercera Edad, para cada categoría de indice retrospectivo de desigualdad

(Españoles mayores de 64 años)

\begin{tabular}{|c|c|c|c|}
\hline & \multicolumn{3}{|c|}{ Indice retrospectivo de desigualdad } \\
\hline & $\begin{array}{c}\text { Estrato rico } \\
\text { (617) }\end{array}$ & $\begin{array}{c}\text { Estrato medio } \\
(1.195)\end{array}$ & $\begin{array}{c}\text { Estrato pobre } \\
\text { (653) }\end{array}$ \\
\hline \multicolumn{4}{|l|}{ Servicios, prestaciones: } \\
\hline 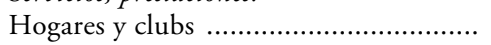 & 93 & 88 & 77 \\
\hline 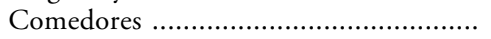 & 61 & 51 & 47 \\
\hline 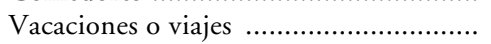 & 89 & 83 & 68 \\
\hline 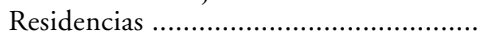 & 86 & 81 & 70 \\
\hline 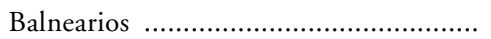 & 74 & 66 & 52 \\
\hline Ayuda a domicilio .................................. & 80 & 67 & 56 \\
\hline 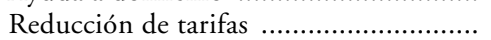 & 82 & 71 & 56 \\
\hline
\end{tabular}

FUENTE: CIS, estudio núm. 2072, 1993 (elaboración propia).

El contraste se produce con gran regularidad y no se nos ocurren comentarios que no resulten ya redundantes. La falta de información, como de actividad, ha de conducir a procesos de aislamiento social.

\section{DESIGUALDAD EN CUANTO A OTROS ASPECTOS PSICOSOCIALES}

Vamos a terminar con unas observaciones basadas en respuestas seleccionadas por los encuestados en torno a aspectos psicológicos y psicosociales de su bienestar. La tabla 9 presenta una aproximación al grado de satisfacción subje-

9 Esta serie de servicios y prestaciones — satélites de la jubilación forzosa- pueden contribuir a acentuar la dependencia estructurada de los ancianos. Lo que ahora interesa no son tanto estas consideraciones críticas como el simple hecho de que los estratos sociales más bajos están en general peor informados, incluso acerca de los servicios y prestaciones que el Estado pone a su disposición. 
tiva de los ancianos con sus relaciones sociales. La gran mayoría de ellos se sienten "muy» o "bastante satisfechos» de sus relaciones con familiares, amigos o conocidos. Son las diferencias entre esas dos categorías — según intervalos de desigualdad retrospectiva controlando por discapacidad alcanzada - las más susceptibles de interpretación.

\section{TABLA 9}

Grado de satisfacción general en sus relaciones con familiares, amigos o conocidos, según indice de discapacidad alcanzada e indice retrospectivo de desigualdad (porcentajes verticales)

(Españoles mayores de 64 años)

\begin{tabular}{|c|c|c|c|c|c|c|}
\hline & \multicolumn{6}{|c|}{$\begin{array}{l}\text { Indice de discapacidad alcanzada } \\
\text { Indice retrospectivo de desigualdad (intervalos operativos) }\end{array}$} \\
\hline & \multicolumn{3}{|c|}{ Capacitados } & \multicolumn{3}{|c|}{ Discapacitados } \\
\hline & $\begin{array}{l}\text { Estrato } \\
\text { rico }\end{array}$ & $\begin{array}{c}\text { Estrato } \\
\text { medio }\end{array}$ & $\begin{array}{l}\text { Estrato } \\
\text { pobre }\end{array}$ & $\begin{array}{l}\text { Estrato } \\
\text { rico }\end{array}$ & $\begin{array}{c}\text { Estrato } \\
\text { medio }\end{array}$ & $\begin{array}{l}\text { Estrato } \\
\text { pobre }\end{array}$ \\
\hline \multicolumn{7}{|l|}{ Satisfacción con sus relaciones: } \\
\hline Muy satisfecho ..................... & 54,1 & 49,1 & 35,5 & 38,5 & 42,2 & 43,1 \\
\hline Bastante satisfecho ................. & 42,6 & 46,4 & 58,5 & 51,0 & 47,8 & 45,6 \\
\hline Poco satisfecho ...................... & 3,0 & 4,0 & 3,6 & 7,5 & 7,2 & 8,1 \\
\hline \multirow[t]{2}{*}{ Nada satisfecho .................. } & 0,3 & 0,4 & 2,3 & 3,0 & 2,8 & 3,2 \\
\hline & $(518)$ & $(868)$ & $(359)$ & $(78)$ & $(256)$ & $(230)$ \\
\hline
\end{tabular}

FUENTE: CIS, estudio núm. 2072, 1993 (elaboración propia).

El porcentaje de «muy satisfechos» disminuye sensiblemente, para los capacitados (que son la mayoría), entre los estratos rico y pobre. Esta desigualdad desaparece, invirtiéndose incluso ligeramente, para la minoría discapacitada. No es sencillo interpretar esto sin una buena dosis de especulación. Lo que nos parece más razonable es plantearlo como un problema de privación relativa. Mientras se encuentran en buenas condiciones físicas, las relaciones sociales de los ricos son más satisfactorias. Cuando llegan a padecer serias discapacidades, sin embargo, es difícil satisfacer a unos individuos acostumbrados a una vida social más placentera y diversa de la que ya no podrán disfrutar. Un tipo así de "pobreza» alcanza a los ricos, pues ellos también envejecen. Los que ya eran pobres, más habituados a toda suerte de padecimientos - en esto sí es más diversa su vida—, soportan mejor la discapacidad y valoran más positivamente el apoyo social que reciben de sus más allegados. 
Junto a la libertad de que puedan gozar, probablemente sea ésta su única "riqueza» ${ }^{10}$.

Otra pregunta de la encuesta del CIS invita a los entrevistados a elegir entre una serie de enunciados dirigidos a averiguar, según su formulación literal, «cuál ha sido su estado de ánimo durante los últimos meses» ${ }^{11}$ La tabla 10

TABLA 10

Estado de ánimo durante los últimos meses según indice de discapacidad alcanzada e indice retrospectivo básico de desigualdad (porcentajes verticales). (Españoles mayores de 64 años)

\begin{tabular}{|c|c|c|c|c|c|c|}
\hline & & $\begin{array}{r}\text { Ind } \\
\text { retrospec }\end{array}$ & $\begin{array}{l}\text { de discap } \\
o \text { de desig }\end{array}$ & $\begin{array}{l}\text { dad alca } \\
\text { ldad (int }\end{array}$ & $\begin{array}{l}\text { ada } \\
\text { alos oper }\end{array}$ & \\
\hline & & Capacitado & & & scapacita & \\
\hline & $\begin{array}{l}\text { Estrato } \\
\text { rico }\end{array}$ & $\begin{array}{c}\text { Estrato } \\
\text { medio }\end{array}$ & $\begin{array}{l}\text { Estrato } \\
\text { pobre }\end{array}$ & $\begin{array}{l}\text { Estrato } \\
\text { rico }\end{array}$ & $\begin{array}{c}\text { Estrato } \\
\text { medio }\end{array}$ & $\begin{array}{c}\text { Estrato } \\
\text { pobre }\end{array}$ \\
\hline 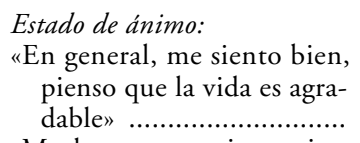 & 74,4 & 66,5 & 58,3 & 40,2 & 40,3 & 30,7 \\
\hline $\begin{array}{l}\text { «Muchas veces me siento triste } \\
\text { y deprimido» ...................... } \\
\text { «Siento que todavía soy útil y }\end{array}$ & 14,9 & 22,6 & 32,3 & 50,7 & 50,6 & 53,7 \\
\hline necesario para la gente» ... & 8,5 & 7,1 & 5,4 & 3,9 & 2,9 & 3,1 \\
\hline «Me siento solo» ....................... & 0,3 & 1,9 & 1,8 & 0,0 & 1,9 & 3,0 \\
\hline «Me siento más irritable» .... & 0,1 & 1,0 & 0,5 & 3,4 & 3,4 & 7,0 \\
\hline $\begin{array}{l}\text { «Me siento con ánimos de em- } \\
\text { prender cosas nuevas»...... }\end{array}$ & 0,9 & 0,6 & 0,2 & 0,9 & 0,0 & 0,0 \\
\hline $\begin{array}{l}\text { "Creo que si desapareciera, na- } \\
\text { die me echaría de menos".. }\end{array}$ & 0,8 & 0,3 & 1,6 & 0,9 & 0,8 & 2,5 \\
\hline & $(513)$ & $(867)$ & $(354)$ & (76) & $(241)$ & $(220)$ \\
\hline
\end{tabular}

FUENTE: CIS, estudio núm. 2072, 1993 (elaboración propia).

${ }^{10}$ Quizá les ocurre a muchos lo que sugiere Sen (1995: 68): «... En situaciones de privación duradera las víctimas (...), muy a menudo, hacen grandes esfuerzos para gozar de los pequeños placeres a su alcance y reducir sus deseos personales a proporciones modestas o realistas». Influyen - claro está- otros factores individuales y culturales. También es posible hablar, como lo hace Moos (1988), de "consecuencias positivas de la adversidad». Hay gente que resiste bien las penalidades. Dice Moss que «ellos pueden salir de una crisis con una mayor confianza en sí mismos, nuevas habilidades para enfrentar otras crisis, relaciones más estrechas con familiares y amigos, y una más positiva apreciación de la vida». Con todo, el propio Moos hace referencia a estos casos como algo fuera de lo normal, aunque sea bastante corriente.

${ }^{11}$ Se les pide que seleccionen un máximo de dos de los enunciados propuestos. En la tabla presentamos únicamente el primero de ellos, ya que sólo el 21,3 por 100 de los encuestados aportó una segunda respuesta. 
muestra la distribución porcentual de respuestas para cada categoría de índice retrospectivo de desigualdad e índice de discapacidad alcanzada.

Los capacitados responden mayoritariamente que, «en general, se sienten bien...", mientras que poco más de la mitad de los discapacitados optan por señalar que «muchas veces se sienten tristes y deprimidos», superando con claridad a los que se sienten bien. Pero las proporciones de los que eligen enunciados con un contenido positivo (como sentirse bien, útil o con ánimos) son siempre mayores entre los ricos que entre los pobres, y viceversa (como sentirse deprimido, solo, irritable o inútil). Este contraste entre ricos y pobres — como ocurría respecto al grado de satisfacción con sus relaciones sociales - tiende a suavizarse cuando se refiere a la categoría de discapacitados. Tal tendencia, de nuevo, puede ser interpretada como un problema de privación relativa. Los pobres - eso parece- soportan mejor que los ricos unas similares adversidades.

Algo parecido ocurre, finalmente, cuando se pide a los jubilados que suscriban o no otra serie de enunciados relativos al momento de su jubilación (Tabla 11) ${ }^{12}$. Todo lo que implica un contenido negativo es afirmado por un porcentaje relativamente mayor entre los más pobres, y viceversa. Son más, por ejemplo, los pobres que «empezaron a tener problemas de salud», «sufrieron periodos de depresión», «se sintieron inútiles» o «tuvieron algunos problemas familiares».

Como única excepción, la jubilación era deseada por algo más del 40 por 100 de los encuestados en todos los casos, sin diferencias notables entre ricos y pobres. Esta «igualdad» podría reflejar el hecho de que la jubilación fue forzosa para casi todos ellos, con escasas diferencias entre los estratos con los que venimos operando.

Destaca, por último, el fuerte contraste entre ricos y pobres — de nuevo a favor de los ricos - respecto al enunciado "pudo hacer cosas (cuando se jubiló) que siempre había querido». Se ha insistido mucho en lo que de positivo tiene, para una buena vejez, la posibilidad de emprender nuevas actividades. La desigualdad hallada concuerda plenamente con la escasez de recursos, de muy diverso tipo, que caracteriza a los estratos sociales más bajos. Estas carencias han de ser causa y efecto de sus menores niveles de actividad social.

${ }_{12}$ No controlamos esta vez por discapacidad alcanzada porque la pregunta está referida al momento de la jubilación, en el cual la capacidad física puede ser muy diferente de la experimentada en el momento de la encuesta. Por otra parte, no incluimos uno de los enunciados de la pregunta, por considerar que responder «sí» o «no» al enunciado literal «No sabía cómo emplear su tiempo" (que es el excluido) induce fácilmente a confusión. 


\section{TABLA 11}

Porcentajes de personas que responden afirmativamente a diversos enunciados relativos a su jubilación, para cada categoría de indice retrospectivo de desigualdad (Españoles jubilados mayores de 64 años)

\begin{tabular}{|c|c|c|c|}
\hline & \multicolumn{3}{|c|}{ Indice retrospectivo de desigualdad } \\
\hline & $\begin{array}{l}\text { Estrato rico } \\
\text { (425) }\end{array}$ & $\begin{array}{l}\text { Estrato medio } \\
\quad(745)\end{array}$ & $\begin{array}{l}\text { Estrato pobre } \\
\text { (304) }\end{array}$ \\
\hline Cuando Vd. se jubiló ... & & & \\
\hline «Tuvo algunos problemas familiares» ... & 1,8 & 1,5 & 4,0 \\
\hline «Lo estaba deseando, se alegró» ............. & 44,5 & 44,3 & 42,3 \\
\hline «Sufrió periodos de depresión» .............. & 10,1 & 11,5 & 15,6 \\
\hline $\begin{array}{l}\text { Pudo hacer cosas que siempre había que- } \\
\text { rido" }\end{array}$ & 36,4 & 34,6 & 18,0 \\
\hline 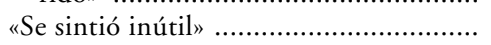 & 8,0 & 11,7 & 12,9 \\
\hline «Empezó a tener problemas de salud» ... & 11,8 & 19,6 & 21,8 \\
\hline
\end{tabular}

FUENTE: CIS, estudio núm. 2072, 1993 (elaboración propia).

\section{UNA VISIÓN GLOBAL DE LOS RESULTADOS}

Tenemos buenas razones para suponer que las desigualdades son más amplias de lo que sugiere esta investigación. Nos hemos ceñido a un universo poblacional relativamente afortunado en términos medios. Dentro de él, es probable que tanto los más ricos como los más pobres escapen en mayor medida a las grandes encuestas. Por otra parte, los intervalos operativos utilizados se han visto parcialmente determinados por imperativos de significatividad estadística. Además, es necesario advertir que hemos trabajado con una muestra de supervivientes a la edad de 65 y más años. En general, las diferencias en renta y condición socioeconómica inciden en la disponibilidad y el uso de los servicios sanitarios, en la morbilidad y en la mortalidad (Rodríguez y De Miguel, 1990: 75-81). Parece claro que sobreviven más ricos que pobres. Esto tiene el lógico efecto estadístico de «enriquecer», en términos medios, cualquier muestra poblacional de supervivientes a determinada edad, sobre todo si ésta es bastante avanzada. Paradójicamente, el que sería más relevante efecto de la pobreza - la muerte física - queda excluido del análisis desde el principio.

Es probable, por otra parte, que entre los más desfavorecidos sobrevivan preferentemente los que gozan de una mayor fortaleza física y/o psíquica o los que mejor han asimilado una subcultura de privación prolongada. Esto tendría el efecto aparente de reducir las desigualdades en cuanto a discapacidad alcanzada debidas a factores socioeconómicos, que se verían compensadas — por confundidas - con las debidas a otros factores tanto de tipo genético como cultural. De haber controlado estos últimos, las desigualdades entre ricos y 
pobres seguramente serían mayores. Todo ello, por cierto, es ilustrativo de las limitaciones propias de una investigación transversal.

Dicho esto, pasamos a proponer una intrepretación global y breve de los resultados de la investigación. Escasa instrucción e ingresos, discapacidad física, aislamiento, falta de actividad social y de información y malestar psicológico, todo ello - entre otras cosas - abierto a interrelaciones y círculos viciosos diversos, son factores más o menos complejos de empobrecimiento. Está en juego, en todo momento, cada proceso real de envejecimiento, susceptible de amplias desigualdades interindividuales. Otras investigaciones han mostrado ya que la salud, la capacidad de adaptación y otros componentes del bienestar se ven sensiblemente afectados por las desigualdades sociales. Lo peculiar de este trabajo no estriba tanto en una serie de resultados numéricos, sino en su interpretación como indicativos de envejecimiento real. Por más que se trate de un proceso universal e irreversible y por fructífera que haya sido alguna ambigua idea de «normalidad» al respecto, no resulta fácil comprender un envejecimiento real ajeno a sus correlatos convencionales (deterioro físico, psíquico y social).

Sugerimos, por tanto, que la mayoría de los individuos situados en los estratos sociales más bajos vienen envejeciendo con relativa mayor rapidez. Acaso empobrecen, más que envejecen, con respecto a los individuos mejor situados social y económicamente. Para comprenderlo, no hay más que pensar en una serie de aspectos vitales como la alimentación, el ejercicio físico e intelectual, los cuidados ante enfermedades, la elección y adecuación de la propia vivienda, etc. En todos los casos y, en definitiva, para una buena calidad de vida y para optimizar el proceso de envejecimiento, es necesario un conocimiento acerca de lo que es mejor, además de otros recursos (dinero, principalmente) que permitan obtener los bienes y servicios de que se trate. Unas desigualdades conducen a otras, con una tendencia general a la acumulación de ventajas por parte de unos y desventajas por parte de otros.

Un buen resumen puede ser presentado señalando que la pobreza influye en muchos de los aspectos que enaltecen diferentes perspectivas teóricas complementarias acerca de la naturaleza biológica, psíquica y social del envejecimiento. La pobreza influye, negativamente en general, en la alimentación y en la aparición y desarrollo de enfermedades; en el desarrollo intelectual, afectivo y emocional; en el aislamiento, el proceso de "disengagement» y la actividad social; en la adaptación a las políticas de vejez y el sometimiento a las consecuencias de la dependencia estructurada.

Abundan los factores incontrolados e incontrolables en cualquier investigación sobre el envejecimiento humano. La expuesta aquí ha mostrado una serie de regularidades en torno a un mayor o más rápido envejecimiento real —un empobrecimiento relativo- por parte de los que fueron y son más pobres. Tales procesos de generación y acentuación de desigualdades son de carácter probable, lo que es fiel y comprensible reflejo de la extraordinaria complejidad de los procesos humanos. 


\title{
BIBLIOGRAFÍA
}

Alfageme, A. (1997): De la pobreza de los viejos, a la vejez de los pobres, Tesis doctoral (próxima publicación por la Universidad de Alicante).

- (1998a): "Velocidad vital de envejecimiento», Revista Multidisciplinar de Gerontología, 8: 73-79.

- (1998b): «La soledad y el género. Una aproximación factorial a la desigualdad económica entre los ancianos españoles de los años noventa», Revista Multidisciplinar de Gerontología (en prensa).

BAZO, M. T. (1990): La sociedad anciana, CIS-Siglo XXI, Madrid.

FERICGLa, J. M. (1992): Envejecer. Una antropología de la ancianidad, Anthropos, Barcelona.

INE (1987a): Encuesta sobre deficiencias, discapacidades y minusvalias, INE, Madrid.

- (1987b): Encuesta sobre deficiencias, discapacidades y minusvalias. Un primer comentario de los resultados, INE, Madrid.

INSERSO (1995): Las personas mayores en España. Perfiles. Reciprocidad familiar, INSERSO, Madrid.

Moos, R. H. (1988): «Life stressors and coping resources influence health and well-being», Evaluación Psicológica, vol. 4, 2.

Rodríguez, J. A., y De Miguel, J. M. (1990): Salud y poder, CIS-Siglo XXI, Madrid.

Rodríguez, J. A. (1994): Envejecimiento y familia, CIS-Siglo XXI, Madrid.

RODRIGUEZ, P. (1995): Introducción, en INSERSO (1995), op. cit.

SÁnchez Vera, P. (1997): "Dimensiones del envejecimiento", Cuadernos de Realidades Sociales, núm. 49150, Instituto de Sociología Aplicada de Madrid.

SEN, A. (1995): Nuevo examen de la desigualdad, Alianza, Madrid.

TorTosA, J. M. (1993): La pobreza capitalista, Tecnos, Madrid.

\begin{abstract}
There is not a satisfactory definition of aging as an universal and irreversible process. Real or observable aging is identificated from his conventional correlates (physical, psychic and social deterioration). Utilizing spanish survey data, we support the hypothesis that the relatively poor people (because their educational and economical status) tend to suffer a worse and quicker process of aging. Most of them reach higher levels of disability, social isolation and psychological discomfort. They make poor, and really make old, in relation to those who have a better social position.
\end{abstract}

